Supplementary Material for

\begin{abstract}
Absolute Rate Constants for Reactions of Tri- $\boldsymbol{n}$-butylstannyl Radicals with Bromoalkanes, Episulfides, and $\alpha$-Halomethyl-Episulfides, -Cyclopropanes and Oxiranes: New Rate Expressions for Sulfur and Bromine Atom Abstraction.
\end{abstract}

James A. Franz", Wendy J. Shaw, Claude N. Lamb, Tom Autrey, Douglas S. Kolwaite, Donald M. Camaioni, and Mikhail S. Alnajjar

Pacific Northwest National Laboratory, 902 Battelle Boulevard, Richland, WA 99352 james.franz@pnl.gov

\title{
TABLE of CONTENTS of SUPPLEMENTARY MATERIAL:
}

Computational Summaries from Gaussian-98 calculations are provided: page:

(a) Vinylthiomethyl radical geometry optimized at UB3LYPl6-311+G(2d,2p) .......S1

(b) Allylthiyl Radical Geometry Optimized at UB3LYPI6-311+G(2d,2p) ............S1

(c) Transition State 1 for C-C Bond Formation of thiiranylmethyl radical, Geometry

Optimized at UB3LYPI6-311+G(2d,2p) .....................................S2

(d) Transition State 2 for Methylene Rotation and C-S scission of thiiranylmethyl

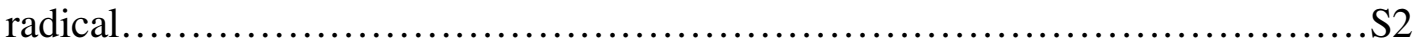

(e) UB3LYP/6-311+G(2d,2p) Analytical Frequencies for Species (a)-(d) above on Vinylthiomethyl $\rightarrow$ Allylthiyl Potential Surface................................S2

(f) Detailed Potential Surface for Vinylthiomethyl—Allylthiyl Rearrangement at

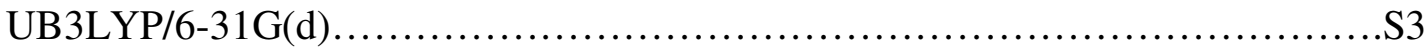

Vinylthiomethyl Radical Geometry Optimized at UB3LYP\6-311+G(2d,2p):

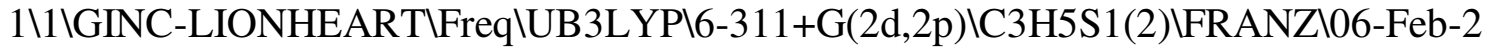
00310\\\#N GEOM=ALLCHECK GUESS=TCHECK UB3LYP/6-311+G(2D,2P) OPT

FREQ SCF=TIGHTIIvinyl thiomethyl

g.s. IV0,2lS, $0.5109360165,0.5450020284,0.2404781581 \backslash \mathrm{C},-1.84$

$16617262,-0.5119243735,0.028315167 \backslash \mathrm{C}, 0.8511646872,-0.3910144314,-0.373$

$1482866 \backslash \mathrm{C}, 2.1222220203,-0.081680968,-0.1313865652 \backslash \mathrm{H},-2.8171428468,-0.1$

$044082048,0.2303666114 \backslash \mathrm{H},-1.6996674042,-1.5654488127,-0.144477758 \backslash \mathrm{H}, 0$.

$5777231114,-1.2305930595,-0.998637473 \backslash \mathrm{H}, 2.9175388312,-0.6648749699,-0$.

$5722385795 \backslash \mathrm{H}, 2.4061746848,0.7530112294,0.4946547782$ IVersion=SGI64-G98

RevA.11\HF=-515.5052333\S2=0.757088\S2-1=0. IS2A=0.750037\

Allylthiyl Radical Geometry Optimized at UB3LYP\6-311+G(2d,2p):

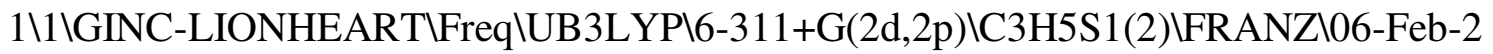


003\0\\#N GEOM=ALLCHECK GUESS=TCHECK UB3LYP/6-311+G(2D,2P) OPT FREQ SCF=TIGHT\lallylthiyl radical $\backslash 0,2 \backslash C,-0.9773784562,-0.2045960396,-$ $1.9993888114 \backslash \mathrm{C},-0.9867362321,-0.1940313012,-0.6714909325 \backslash \mathrm{S}, 1.0266238902,-$ $0.2023548901,1.2439198787 \backslash \mathrm{C},-0.126748841,0.7033278688,0.1604585875 \backslash \mathrm{H},-$ $0.7324002074,1.2860975526,0.8644809442 \backslash \mathrm{H}, 0.4290284593,1.4153417156,-$ $0.4480771232 \backslash \mathrm{H},-1.6297001601,-0.8744455827,-0.1253824284 \backslash \mathrm{H},-1.6144643967,-$ $0.8718392398,-2.5627605027 \backslash \mathrm{H}, 0.3332647626,0.4543206286,2.5684520103$

IIVersion=SGI64- G98RevA. $11 \backslash \mathrm{HF}=-515.522153 \backslash \mathrm{S} 2=0.753577 \backslash \mathrm{S} 2-1=0 . \mid \mathrm{S} 2 \mathrm{~A}=0.750006 \backslash$

\section{Transition State 1 for C-C Bond Formation, Geometry Optimized at UB3LYP|6-} $311+G(2 d, 2 p)$ :

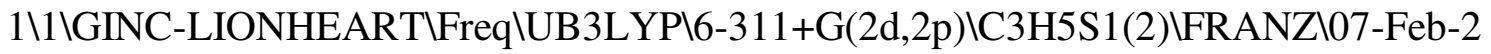
003\0\\B3LYP/6-311+G(2D,2P) OPT=TS FREQ GEOM=CHECK GUESS=READ $\mathrm{SCF}=\mathrm{TIGHT} \backslash \backslash$ thiiranylmethyl ts $\backslash \backslash 0,2 \backslash \mathrm{C},-0.7294598426,-0.3555466452,-1.6846453433 \backslash$ C,-0.7296403036,-0.3541973985,-0.3179204413।S,0.7492776975 ,$0.3509199201,0.683502632 \backslash \mathrm{C}, 0.0250607747,1.2526948753,0.6561234681 \backslash \mathrm{H},-$ $0.6306931031,1.5610624473,1.4561924921 \backslash \mathrm{H}, 0.3728355082,1.9721503431,-0$ $.0671850362 \backslash \mathrm{H},-1.6484114288,-0.5034509799,0.230507283 \backslash \mathrm{H},-1.6652247543,-$ $0.3800992473,-2.2253322994 \backslash \mathrm{H}, 0.1872868468,-0.2926488316,-2.2515706521$

IIVersion=SGI64-G98RevA.11 $\backslash \mathrm{HF}=-515.4725644 \backslash \mathrm{S} 2=0.778949 \backslash \mathrm{S} 2-1=0 . \backslash \mathrm{S} 2 \mathrm{~A}=0.75018 \backslash$

\section{Transition State 2 for Methylene Rotation and C-S scission.}

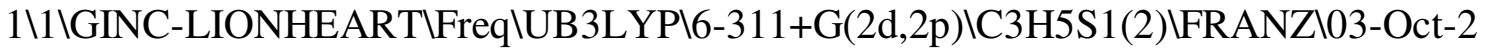
003\0\\#B3LYP/6-311+G(2D,2P) FREQ GEOM=CHECK GUESS=READ $\mathrm{SCF}=\mathrm{TIGHT} \backslash \mathrm{TS}$

for methylene rotation of vtm radical opening $\backslash \backslash 0,2 \backslash \mathrm{C},-0.4779328265,0.5$ 97794582,0.0549137018\C,1.0011760702,0.6261006978,0.2169797622\S,0.397 $2919505,-0.8045266302,-0.763748382 \backslash C,-1.3608487304,0.3288971889,1.1994$ 455706\H,-0.8608269241,1.3086357539,-0.674188855\H,1.567891574,1.35810 $00487,-0.3416144156 \backslash \mathrm{H}, 1.40171811,0.3696555676,1.1874716086 \backslash \mathrm{H},-1.470555$ $745,1.079297194,1.9715340427 \backslash \mathrm{H},-1.9692653032,-0.5600172931,1.248737523$ $7 \backslash$ Version=SGI64-G98RevA.7\HF=-515.4960473 $\backslash S 2=0.753828 \backslash \mathrm{S} 2-1=0 . \backslash \mathrm{S} 2 \mathrm{~A}=0.7$ $50009 \backslash$

UB3LYP/6-311+G(2d,2p) Frequencies for Species on Vinylthiomethyl $\rightarrow$ Allylthiyl Potential Surface 


$\begin{array}{cccc}\text { Allylthiyl } & \text { Vinylthiomethyl } & \text { TS1 } & \text { TS2 } \\ 92.7728 & 56.4969 & -551.7712 & -271.2898 \\ 272.0001 & 208.0567 & 263.611 & 300.5709 \\ 402.3885 & 238.7176 & 367.449 & 367.0047 \\ 591.7352 & 322.7363 & 501.306 & 520.2712 \\ 704.541 & 414.0674 & 512.656 & 599.6979 \\ 790.0682 & 587.5662 & 673.673 & 635.6318 \\ 736.9567 & 690.6097 & 707.771 & 886.2136 \\ 953.8749 & 834.8957 & 804.982 & 924.8631 \\ 1021.534 & 914.9785 & 806.88 & 975.1918 \\ 1069.301 & 934.8337 & 932.796 & 1052.2 \\ 1180.14 & 983.9038 & 953.347 & 1072.815 \\ 1241.268 & 1054.862 & 1041.66 & 1110.472 \\ 1326.465 & 1295.753 & 1265.24 & 1183.517 \\ 1401.018 & 1405.46 & 1332.69 & 1336.097 \\ 1459.595 & 1423.769 & 1419.47 & 1445.8 \\ 1694.223 & 1635.415 & 1501.88 & 1483.393 \\ 2998.577 & 3148.979 & 3137.34 & 3075.527 \\ 3076.557 & 3170.645 & 3146.72 & 3215.229 \\ 3137.164 & 3172.329 & 3179.62 & 3139.421 \\ 3157.231 & 3235.438 & 3243.69 & 3215.971 \\ 3223.115 & 3299.416 & 3266.94 & 3253.215\end{array}$

Potential Surface for Vinylthiomethyl-Allylthiyl Rearrangement at UB3LYP/6$31 \mathrm{G}(\mathrm{d}) 0^{\circ} \mathrm{K}$ Electronic energies, hartree/particle

$\begin{array}{cc}\text { AngleC2-S3-C4 } & \text { Energy, hartree/particle } \\ 30 & -515.45164 \\ 35 & -515.45117 \\ 40 & -515.44795 \\ 45 & -515.4414 \\ 50 & -515.43563 \\ 55 & -515.4255 \\ 60 & -515.41374 \\ 65 & -515.4041 \\ 70 & -515.40032 \\ 75 & -515.40253 \\ 80 & -515.40877 \\ 85 & -515.41694 \\ 90 & -515.42323 \\ 95 & -515.42736 \\ 100 & -515.42933 \\ 105 & -515.42935\end{array}$

